\title{
GESTIÓN UNIVERSITARIA. CONTRIBUCIÓN DEL ESTUDIANTE PARA EL PROCESO DE EVALUACIÓN DE DESEMPEÑO DOCENTE, APLICACIÓN EN LA UNIVERSIDAD JOSÉ EDUARDO DOS SANTOS, HUAMBO-ANGOLA
}

\author{
Helder Sntos Cangovi \\ Universidade José Eduardo dos Santos (Angola) \\ cangovi@gmail.com
}

\begin{abstract}
Resumen. La gestión de personas se ha convertido en un importantísimo instrumento para la garantía de la supervivencia de las organizaciones y, las instituciones de enseñanza superior no están ajenas a esta realidad. Así, la gestión universitaria, se caracteriza por la implicación de los más variados elementos integrantes de la misma (estudiantes, docentes, gestores, sociedad), debido a su extensión de productividad. La presente investigación tiene como principal objetivo contribuir a mejorar la gestión universitaria en la Universidad José Eduardo dos Santos (UJES) mediante la participación de los estudiantes en el proceso de evaluación del desempeño de los docentes, proponiendo de ese modo un procedimiento de evaluación que integra la participación de los estudiantes en el programa. Para ello se realizó una revisión bibliográfica sobre las diferentes contribuciones de diversos autores, desde el punto de vista de la gestión general para la gestión de Recursos Humanos y de éste para la evaluación del desempeño docente. Se constituyó para el trabajo un estudio de caso aplicado en la UJES, ubicada en la $V$ región académica, en Angola. De esta forma, para el logro de los objetivos de esta investigación se elaboró una encuesta destinada a los estudiantes que permitió concluir que la participación de los estudiantes en el proceso de evaluación de desempeño docente juega un papel importantísimo, ya que ellos conviven directamente con el docente en el aula y, que la adopción de un modelo de evaluación que permita la participación de éstos puede contribuir a la mejora de la calidad de la enseñanza y el aprendizaje, y con ello la mejora de la gestión institucional.
\end{abstract}

Palabras clave: Gestión Universitaria, Recursos Humanos, Evaluación de Desempeño, Estudiante, Docente Universitario. 


\title{
GESTÃO UNIVERSITÁRIA. CONTRIBUIÇÃO DO ESTUDANTE PARA O PROCESSO DE AVALIAÇÃO DE DESEMPENHO DOCENTE, APLICAÇÃO NA UNIVERSIDADE JOSÉ EDUARDO DOS SANTOS, HUAMBO-ANGOLA
}

\begin{abstract}
Resumo. A gestão de pessoas se tem convertido num importantíssimo instrumento para o garante da sobrevivência das organizações e as instituições de ensino superior não estão alheias a esta realidade. Assim, a gestão universitária, se caracteriza pelo envolvimento dos mais variados elementos integrantes da mesma (estudantes, docentes, gestores, sociedade), isto devido a sua extensão de produtividade. A presente pesquisa tem como principal objectivo contribuir para melhorar a gestão universitária na Universidade José Eduardo dos Santos (UJES) mediante a participação dos estudantes no processo de avaliação do desempenho dos docentes, propondo desse jeito um procedimento de avaliação que integra a participação dos estudantes no mesmo processo. Para tal foi feita uma revisão bibliográfica sobre os diferentes contributos de diversos autores desde o ponto de vista da gestão geral para a gestão de Recursos Humanos e deste para a avaliação de desempenho docente. Constituiu-se ainda para o trabalho um estudo de caso aplicado na UJES, localizada na $\mathrm{V}$ região académica, em Angola. Desta forma, para o alcance dos objectivos desta investigação foi elaborado um inquérito destinado aos estudantes que permitiu concluir que a participação dos estudantes no processo de avaliação de desempenho docente, joga um papel importantíssimo, visto que eles convivem directamente com o docente na sala de aulas e que a adoção de um modelo de avaliação que permita a participação destes pode contribuir para a melhoria da qualidade de ensino e aprendizagem e com isto no melhoramento da gestão institucional
\end{abstract}

Palavras-chave: Gestão Universitária, Recursos Humanos, Avaliação de Desempenho, Estudante, Docente Universitário.

\section{UNIVERSITY MANAGEMENT. CONTRIBUTION OF THE STUDENT TO THE PROCESS OF EVALUATION OF TEACHING PERFORMANCE, AT THE JOSÉ EDUARDO DOS SANTOS UNIVERSITY, HUAMBO-ANGOLA}

\begin{abstract}
People management has become a very important instrument for guaranteeing the survival of organizations and institutions of higher education are not unawareof this reality. Thus, university management is characterized by the involvement of the most diverse elements (students, teachers, managers, and society), due to its extension of productivity. The main objective of this research is to contribute to the improvement of university management at the University José Eduardo dos Santos (UJES) through the participation of students in the evaluation process of teachers 'performance, thus proposing an evaluation procedure that integrates students' participation in the same process. For this, a bibliographic review was done on the different contributions of several authors from the point of view of the general management for Human Resources management and from this point to the evaluation of teaching performance. A case study was also carried out at UJES, located in the fifth academic region, in Angola. Thus, in order to reach the objectives of this research, a survey was carried out for students that allowed the conclusion that the participation of students in the evaluation process of teaching performance plays a very important role, since they live directly with the teacher in the classroom and that the adoption of an evaluation model that allows the participation of the latter can contribute to the improvement of the quality of teaching and learning and with this in the improvement of institutional management
\end{abstract}

Key words: University Management, Human Resources, Performance Evaluation, Student, University Teacher. 


\section{Introducción}

Todos los días se verifica a nivel mundial cambios en la actuación de los profesionales a nivel laboral. Estos cambios exigen nuevos paradigmas en la actuación de los colaboradores y de la participación de los distintos agentes involucrados en el proceso de evaluación del desempeño de estos profesionales. De este modo, cada individuo en el uso de sus funciones adopta conductas para enfrentar mejor los retos de su área de actuación y estos no escapan de las instituciones educativas que tienen la misión de potenciar los procesos de formación humana y profesional, de manera que estos tengan competencias para enfrentar con éxito los retos de hoy y de mañana en un mundo cada vez más competitivo.

Siendo así, la evaluación de los docentes se convirtió en una herramienta muy útil en las instituciones educativas, pues permite tomar las decisiones más diversas en el sentido de mejorar la cualidad tanto de los docentes mismos y del proceso de enseñanza-aprendizaje y, así, la calidad de los alumnos.

De los estudios previos realizados, se encontró que la evaluación de desempeño docente en la Universidade José Eduardo dos Santos (UJES) se basa de manera genérica en el Decreto n..$^{\circ}$ 25/94, del 1 de Julio, cuyo procedimiento es lo mismo hacia todos los funcionarios públicos (Fernandes, 2014). Según este autor, el procedimiento no se ajusta a la realidad del profesional de educación superior angoleña $\mathrm{y}$, de hecho, este ha sido una preocupación de las autoridades de la enseñanza superior en el país, de manera que esta herramienta de evaluación del desempeño permite resaltar algunas calidades muy generales, que sirven para evaluar el desempeño de cualquier funcionario público y que no se ajustan a la realidad educativa.

El presente trabajo tiene como su marco de aplicación la UJES, institución pública de educación superior angoleña creada en 2009, mediante el Decreto 7/09, de 12 de mayo, siendo que las normas vigentes son las mismas que las vigentes en la Universidade Agostinho Neto, en aquél momento la única en el país. Con respecto al enfoque, el procedimiento utilizado para medir el desempeño docente en la UJES no tiene en cuenta una perspectiva de evaluación de 360 grados, es decir, todos los actores involucrados en la enseñanza, con énfasis en los estudiantes mientras elemento principal, que en su cuotidiano se relacionan con estos.

De esta manera, el problema de esta investigación estuvo relacionado con la contradicción entre la necesidad de evaluar periódicamente el desempeño de los docentes de la Universidade José Eduardo dos Santos y la necesidad de involucrar la participación de los estudiantes en el mismo proceso como una forma de detectar las principales debilidades y mejorar las habilidades de trabajo, las que permiten un desempeño individual y organizacional superior. Desde esta perspectiva, se obtuvo la siguiente cuestión científica: ¿Cómo podría mejorarse el desempeño de los docentes de la UJES mediante la participación del estudiante en el proceso de evaluación del desempeño docente, de manera a contribuir a la gestión universitaria? Dada la pregunta científica, se derivó la siguiente hipótesis:

Si se garantiza a los estudiantes la participación en el proceso de evaluación del desempeño docente, podrían estos contribuir a mejorar el desempeño de la Universidad José Eduardo dos Santos.

De esta manera, dada la hipótesis anterior, se presentan los siguientes objetivos: 


\section{Objetivo general}

Contribuir a mejorar la gestión universitaria en la UJES a través de la participación de los estudiantes en el proceso de evaluación del desempeño docente.

\section{Objetivos específicos}

i) Teóricamente apoyar la gestión, desde el punto de vista de la gestión universitaria y del proceso de evaluación del desempeño de la enseñanza de Recursos Humanos, ii) diagnosticar el estado actual de la evaluación del desempeño de los docentes de la UJES y la participación de los estudiantes en este proceso y iii) proponer un procedimiento y un respectivo modelo que permita a los estudiantes participar en el proceso de evaluación del desempeño del profesorado de la UJES.

\section{Objeto, ámbito y horizonte de investigación.}

En vista del enfoque presentado anteriormente, se evidencia que el estudio se desarrolla desde la gestión de manera general hasta la gestión universitaria y, como ámbito de estudio, de la gestión de los Recursos Humanos hasta la evaluación del desempeño dentro de la universidad, desplegándose en cascada como se presenta en la figura 1:

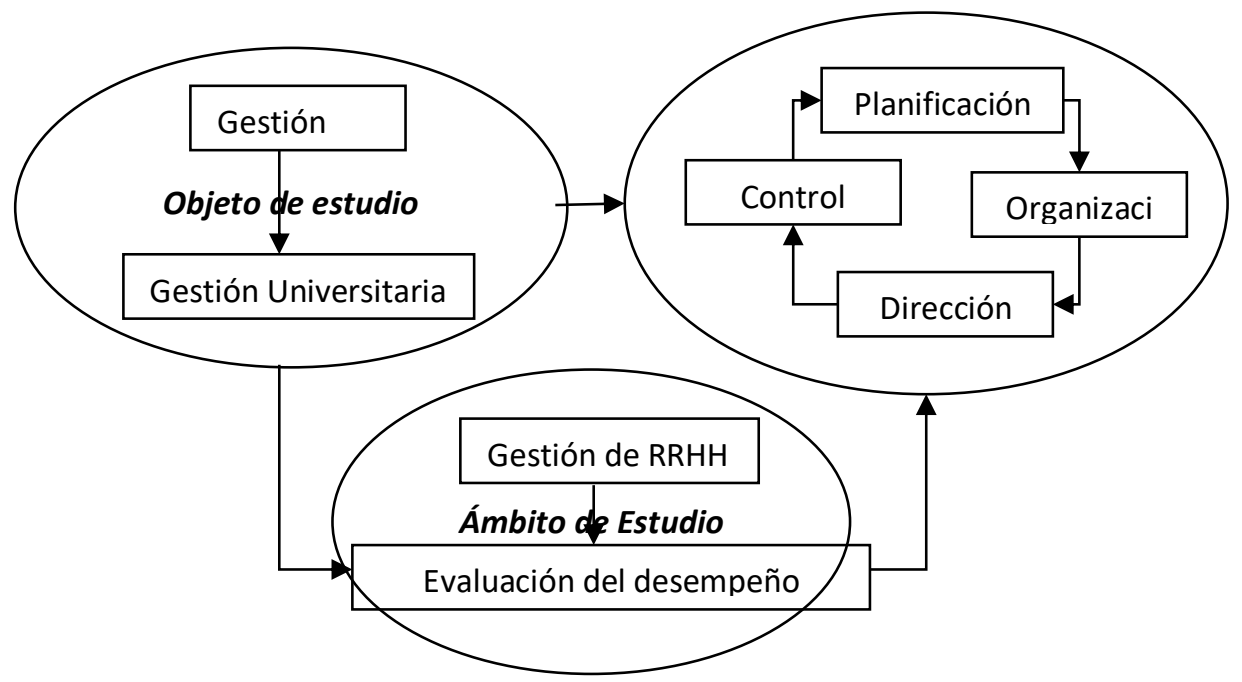

Figura 1. Objeto y ámbito de estudio

Nota: Fuente: el autor

\section{Fundamentos de la gestión universitaria}

Las personas son el motor que impulsa cualquier actividad, son el alma y la sangre de las organizaciones. Se convierten en el centro de todas las organizaciones, sean rentables o no, tales como escuelas, hospitales, centros de rehabilitación, organizaciones no gubernamentales (ONG's), iglesias, universidades, cooperativas y otros, pues constituyen el recurso vivo de cualquier organización y, por señal, el único (Chiavenato, 2009).

La sociedad actual se está volviendo cada vez más exigente por la manera en que se imparte la enseñanza, pero también por la forma en que se administran las universidades. Tal situación va mucho más allá de lo que son meras falacias y de la realidad. La gestión universitaria tiende a ser más exigente por el hecho de que es en las universidades donde se capacitan los distintos gestores de cualquier organización. 
Toda una organización necesita de la existencia de recursos y de personas, ya que en la era actual las personas constituyen el elemento principal de las organizaciones, pues poseen el mayor recurso de la organización, que es el capital humano. La existencia de personas y recursos en la organización permite que se gestione los mismos para que se pueda alcanzar los objetivos deseados.

En un universo cada vez más global, podemos encontrar distintas organizaciones, tanto que las personas pasan la mayor parte del tiempo en ellas, teniendo en cuenta que es en las organizaciones donde los individuos pueden lograr alcanzar objetivos, cosas que nunca podrían lograr de forma aislada o individual. Las organizaciones son de una gran importancia, ya que su rol va más allá que las pretensiones de sus gerentes.

Daft (2010) considera que una organización es una entidad social orientada a metas y estructurada deliberadamente. Para él, una organización es deliberadamente estructurada por el hecho de que se subdivide en partes o distintos y diferentes departamentos.

Cada organización, independientemente de su propósito, necesita administrar sus recursos para lograr sus objetivos.

De este modo, sin la organización, según Drucker (2010), no habría administración, pero sin administración solo podría haber una masa de personas, no una organización. La organización misma es un órgano de la sociedad y existe solo para cooperar con la sociedad, la economía, las personas, aportando el resultado necesario.

La gestión puede definirse como el proceso de lograr resultados (bienes o servicios) con los esfuerzos de otros (Teixeira, 2013). Por otro lado, Tamo (2006) menciona que la gestión es el proceso destinado a obtener resultados utilizando todos o parte de los recursos de la organización.

Para que las organizaciones logren resultados, es necesario que alguien con habilidades pueda administrarlas: a este individuo se le llama gerente, cuyo propósito es contribuir al desempeño de la organización, planeando, organizando y controlando toda actividad de la organización.

\section{La gestión universitaria dentro del ámbito general de la gestión. Los gerentes universitarios}

Según Tosta et al., (2011) la universidad es una institución que posee el conocimiento como materia prima, que este existe para apalancar la sociedad y contribuir para su desarrollo, con el objetivo de formar profesionales calificados.

Gomes et al., (2012) consideran que las instituciones de enseñanza universitaria, para consolidarse como una institución de excelencia en la enseñanza, la investigación y la extensión y tener reconocimiento por la comunidad científica y la sociedad, a nivel nacional e internacional, deben llevar a sus gerentes a preocuparse por los procesos de evaluación [...] que colocan a la institución en una posición de visibilidad, con las clasificaciones específicas de los cursos, y muchas otras formas de mensurar la calidad.

La gestión universitaria debe basarse en los supuestos de una gestión eficiente y eficaz, capaz de dar credibilidad a lo que está orientada, preparada a los desafíos del milenio.

\section{La gestión de los recursos humanos dentro de la gestión universitaria Papel de la evaluación del desempeño de los docentes universitarios.}

Toda organización sobrevive gracias a las personas. En las universidades, donde el capital más grande es el capital humano, se verifica la valoración del hombre. 
Para Camara et al., (2010), el reconocimiento de que no es suficiente disponer de la más avanzada tecnología, de una sólida base financiera o una posición dominante en el mercado para garantizar el éxito, si las mismas no son sostenidas por una fuerza motivada de trabajo, ante a un perfil de habilidades con visión de futuro y alta productividad, constituye un cambio notable en la mentalidad de muchos gerentes.

La gestión debe concebir dentro del ámbito de la organización supuestos que garanticen y reconozcan el valor de la fortaleza humana como un factor crítico del éxito de la organización. Aun así, la valoración de la fuerza humana debe basarse en una visión de futuro, en la concepción de métodos que incluyan en su actuación la gestión participativa, para que los empleados se sientan verdaderos participes en el logro del éxito de la organización, teniendo en cuenta que también tienen sus objetivos individuales.

La gestión de RR.HH. debe basarse en herramientas que se ajusten a un flujo de trabajo que garantice una gestión eficiente, como ilustrado en la figura 2 a continuación:

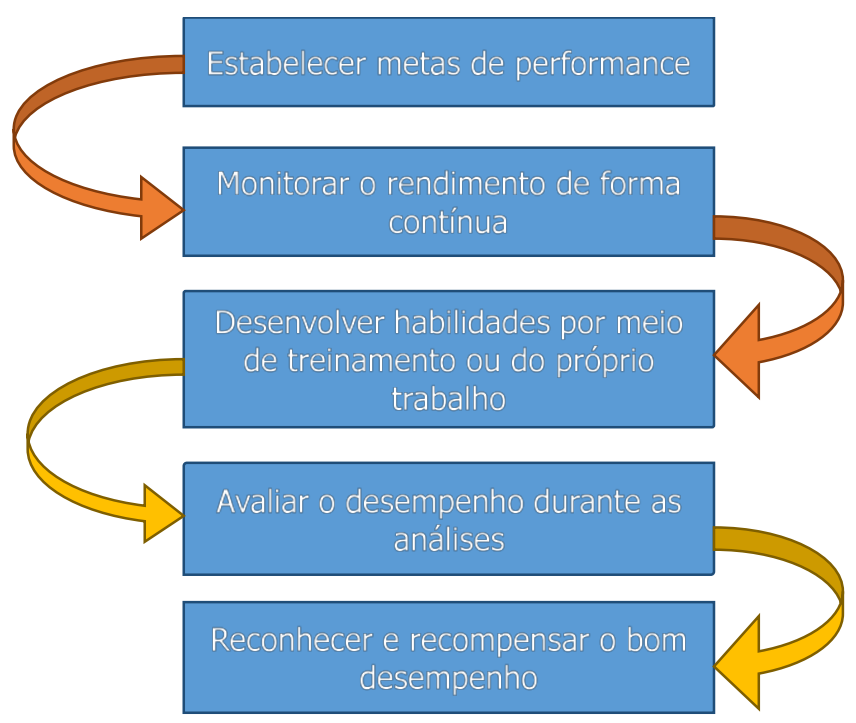

Figura 2. Herramienta de flujo de trabajo a una gestión de desempeño eficiente

Nota: el autor, adaptado de Silverstein (2011)

La evaluación del desempeño en esta situación juega un papel diferenciador y crucial para obtener resultados, comparándolos con los recomendados.

De manera tradicional, la evaluación del desempeño, según Silverstein (2011), significa lo mismo que una valoración de un empleado o «la crítica de su desempeño». Hoy en día, sin embargo, muchas organizaciones van más allá de solo medir el desempeño de sus empleados, adoptando un proceso de gestión del desempeño más integral.

Actualmente, la evaluación de desempeño se centra más en el empleado, en identificar mediante resultados, fortalezas y debilidades, dirigiéndolos hacia la continuación, el desarrollo o la superación. Específicamente, la evaluación de desempeño tiene como objetivo principal el desarrollo del empleado y mediante del cual se puedan lograr los objetivos de la organización. 


\section{Objetivos de la evaluación de desempeño de los docentes}

Pereira (2009), afirma que los procesos de evaluación permiten a los trabajadores saber qué se espera de ellos, los criterios de evaluación de su desempeño, comentarios acerca de la ejecución de su trabajo y la posibilidad de mejorar su desempeño.

Autores como Cassettari (2014), Fernandes (2008) y Rocha (2004) agrupan los objetivos de evaluación docente en dos categorías:

- La que privilegia la función formativa de la evaluación y el desarrollo profesional de los docentes mediante evaluaciones con consecuencias débiles;

- La que privilegia la función sumativa de la evaluación y la responsabilidad de los docentes, a través de evaluaciones con altas consecuencias.

Tabla 1.

Evaluación de naturaleza formativa Vs evaluación de naturaleza sumativa.

\section{Evaluación de naturaleza formativa visa}

Proporcionar retroalimentación de las informaciones e identificar

fortalezas/debilidades individuales

\section{Evaluación de naturaleza sumativa}

visa

Proporcionar retroalimentación de las
informaciones Documentar las decisiones personales
fortalezas/debilidades individuales

Reconocer el desempeño individual y Definir los candidatos a promoción, ayudar a identificar objetos transferencias y asignaciones.

Evaluar el logro de los objetivos e Identificar el bajo rendimiento y decidir identificar las necesidades de capacitación si desea mantener o rescindir los contratos. individual y organizacional.

Reforzar la estructura de la autoridad de selección

Decidir los despidos y validar criterios

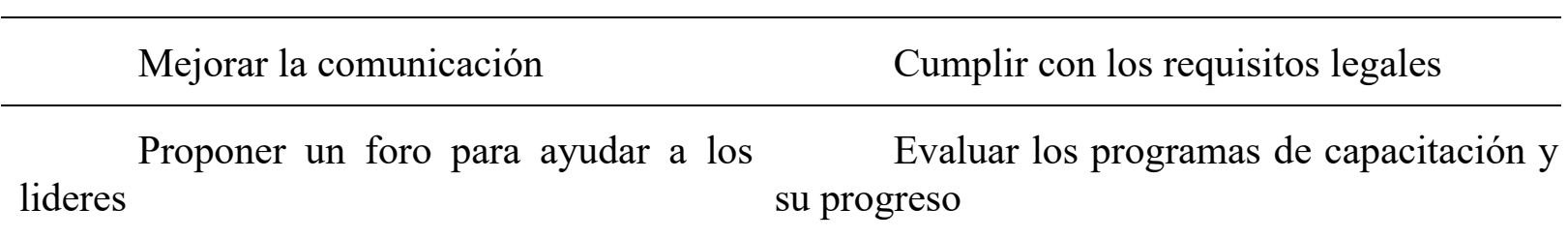
inquietudes

Planificación del personal y toma de decisiones sobre recompensas/remuneración

Nota: adaptado de Bohlander \& Snell (2009)

\section{Métodos modernos de evaluación de desempeño}

Los métodos modernos de evaluación del desempeño, según Carvalho et al. (2012), apuntan una tendencia de autoevaluación de la participación de los empleados involucrados en la planificación de su desarrollo profesional, destacando el futuro en constante mejora de la función de desempeño. 
Los más recientes métodos buscan atender las exigencias organizaciones, así que tenemos: métodos de participación por objetivos, método de evaluación por competencias y el método de evaluación de 360 grados.

Santo \& Santos (2010), afirman que entre las diversas tipologías usadas para evaluar de desempeño de las personas, la evaluación de 360 grados ha cobrado mayor importancia en el entorno organizacional, debido a su posibilidad de escuchar a las partes interesadas, ya que busca desarrollar las competencias a través de la retroalimentación de los compañeros y el propio evaluado. Para estos autores, la evaluación de desempeño de 360 grados se basa en comportamientos observados, es decir, en las percepciones que las personas tienen acerca de determinados comportamientos del empleado evaluado. Los métodos de evaluación de desempeño modernos proporcionan informaciones más convencionales, que reflejan el desempeño del empleado en una determinada organización.

Los principales beneficiarios del sistema de evaluación de desempeño de los docentes a través del método de 360 grados según Pinto \& Oliveira (2005) son:

- La institución de enseñanza superior. Mediante el establecimiento de mecanismos que garanticen la calidad en la enseñanza y promuevan su mejora;

- El docente. El método de evaluación de desempeño garantiza la igualdad en la evaluación, y también incluye la inclusión de factores que van más allá de la docencia y que normalmente no se tienen en cuenta;

- Los estudiantes. Aunque de manera indirecta, y como consecuencia de los dos puntos anteriores, los alumnos resultan ser los principales beneficiarios (mejor enseñanza, mejora de la calidad y docentes más motivados).

La participación de los estudiantes en el proceso de evaluación docente tiene grande relevancia, ya que los mismos lidian directamente con el docente, tanto en el salón de clase como en los trabajos de investigación. El estudiante está preparado para opinar sobre el desempeño del docente cuanto a algunos aspectos, como la interacción estudiante-docente, la actualidad de los temas dados, el tipo de contenido y la capacidad de respuesta a las dudas de los estudiantes.

Sin embargo, para Fernandes, Martinez y Sanchez, (2015), el docente en su desempeño debe obligatoriamente usar todas sus habilidades pedagógicas, didácticas, científicas e interpersonales, desarrollando acciones ajustadas a los aspectos cognitivos de los estudiantes.

De este modo, Cassettari (2014), afirma que los estudiantes experimentan diariamente el trabajo de sus profesores y, por lo tanto, pueden hacer importantes aportes a su evaluación, especialmente en la educación superior, donde puede ser muy útil.

Para Fernandes (2014), el proceso de evaluación de desempeño docente en las instituciones de educación superior públicas angoleñas debe apoyarse en indicadores de desempeño basados en competencias unidas a actividad docente.

\section{Metodología}

Para describir el proceso de evaluación del desempeño, este trabajo tuvo como objetivo elaborar un estudio de caso en la Universidade José Eduardo dos Santos, ubicada en la V Região Acadêmica, creada por el Decreto $\mathrm{N}^{\circ} 5 / 09$, de 7 de abril, del Conselho de Ministros, congregando las provincias de Huambo, Bié y Moxico. 
El objetivo general de la UJES es la capacitación de personal técnico calificado en las más distintas áreas del saber a las que están dedicados, dotados de conocimientos científico y técnico. Actualmente en la UJES se ofrecen 29 cursos de graduación y cinco cursos de maestría, este último en colaboración con las Universidades de Lisboa, Autónoma de Barcelona y Técnica de Lisboa. La institución cuenta con la colaboración de docentes y personal administrativo, cuyo $n .^{\circ}$ se encuentra en la tabla a continuación:

Tabla 2.

Personal de la UJES en 2014

\begin{tabular}{|c|c|c|c|}
\hline & Efectivos & Colaboradores & Total \\
\hline Docentes & 196 & 385 & 581 \\
\hline Personal administrativo & 291 & 106 & 397 \\
\hline Total & 487 & 491 & 978 \\
\hline
\end{tabular}

Nota: retirada del informe de la Universidade José Eduardo dos Santos (2015)

\section{Métodos teóricos de investigación}

Métodos teóricos cumplen una función que posibilita la interpretación conceptual de los métodos empíricos (Sá-Silva et al., citados por Vilelas, 2009). Desde el punto de vista teórico, se utilizan elementos del método dialéctico y sus categorías, tales como: análisis y síntesis, lógico-histórico e inducción - deducción. Integrándose así en el método hipotético deductivo como parte del método científico utilizado en esta investigación.

\section{Métodos empíricos de investigación}

Análisis de documentos: se procuró revisar las diferentes leyes bajo las cuales se apoyan la actividad docente en Angola, a saber:

- La Ley 13/01, de 31 de Diciembre, Ley de Base del Sistema Educativo;

- El Decreto 25/94, de 1 de Julio, procedimiento para la evaluación de desempeño de los funcionarios públicos;

- Directrices para la mejora de la gestión del subsistema de la enseñanza superior;

- Otros documentos directrices sobre el proceso de evaluación del desempeño vigente en la UJES, así como la bibliografía disponible en artículos y trabajos de investigación en diferentes áreas del conocimiento.

Encuestas: conjunto de preguntas formuladas para obtener información que se basa en las respuestas que ciertas personas pueden dar, lo que permite hacer un diagnóstico sobre la situación actual del estado de la evaluación del desempeño docente.

Estadística descriptiva: para caracterizar el comportamiento de los indicadores definidos en la muestra de los estudiantes.

Se envió un cuestionario a los estudiantes de la UJES, siendo que como opciones de respuestas, se utilizó una escala de Likert con 6 puntos: 1 - totalmente en desacuerdo, 2 elevado desacuerdo, 3 - ligeramente en desacuerdo, 4 - de acuerdo, 5 - muy de acuerdo, 6 completamente de acuerdo.

La población de estudio o universo, que es el conjunto de todos elementos, individuos u objetos, que poseen una cierta característica en común (Tamo, 2012), consistió en 10,947 estudiantes matriculados en todas U.O's de la UJES en 2014, según la siguiente tabla 3: 
Tabla 3

Estudiantes de la UJES, matriculados en 2014

\begin{tabular}{ll}
\hline Unidad Orgánica UJES & N. ${ }^{\circ}$ de Estudiantes \\
\hline Facultad de Ciencias Agropecuarias & 665 \\
\hline Facultad de Derecho & 1,152 \\
\hline Facultad de Economía & 1,750 \\
\hline Facultad de Medicina & 457 \\
\hline Facultad de Medicina Veterinaria & 364 \\
\hline Instituto Politécnico Superior del Huambo & 3,285 \\
\hline Escuela Politécnica Superior de Bié & 1,288 \\
\hline Escuela Politécnica Superior de Moxico & 1,986 \\
\hline TOTAL & 10,947 \\
\hline
\end{tabular}

Nota: Fuente: Retirada del Informe de la Universidade José Eduardo dos Santos (2015).

Mediante el procedimiento normal de cálculo de la muestra y considerando que la población es finita, los datos resultaron en una muestra de 372 estudiantes que representan parte de la población, con un nivel de confianza del 95\% y un margen de error del 5\%. De los cuestionarios distribuidos, 342 estudiantes los respondieron, representando el $91.94 \%$ de la muestra, un número que se consideró suficiente para aplicar el estudio bajo análisis.

Los datos se recolectaron mediante la distribución de cuestionarios y de la propuesta de la metodología de aplicación participativa a los estudiantes, después de explicaciones sobre la forma de completar y acerca de los objetivos del cuestionario respectivo. Todas las dudas sobre la realización de los cuestionarios se aclararon en el lugar de entrega.

Tras aproximadamente cuatro semanas, lo que permitió a los encuestados comprender mejor las preguntas formuladas en la encuesta y la propuesta de participación de los estudiantes en el proceso de evaluación docente, los cuestionarios fueron devueltos para su análisis.

Los cuestionarios se distribuyeron al azar a los estudiantes de todas las unidades organizativas de la UJES, como presentado en la tabla 4 a continuación. 
Tabla 4

Número de Cuestionarios respondidos por Estudiantes por institución

\begin{tabular}{ccccc}
\hline Unidad Orgánica & \multicolumn{5}{c}{ Número de Estudiantes } \\
\cline { 2 - 6 } & $\begin{array}{l}\text { N. }{ }^{\circ} \text { de } \\
\text { estudiantes } \\
\text { distribuidos }\end{array}$ & Porcentaje & $\begin{array}{l}\text { Porcentaje } \\
\text { válido }\end{array}$ & $\begin{array}{l}\text { Porcentaje } \\
\text { acumulado }\end{array}$ \\
\hline $\begin{array}{c}\text { Escuela Politécnica Superior de } \\
\text { Bié }\end{array}$ & 58 & 17,0 & 17,0 & 17,0 \\
\hline $\begin{array}{c}\text { Escuela Politécnica Superior de } \\
\text { Moxico }\end{array}$ & 46 & 13,5 & 13,5 & 30,4 \\
\hline $\begin{array}{c}\text { Facultad de Ciencias } \\
\text { Agropecuarias }\end{array}$ & 26 & 7,6 & 7,6 & 38,0 \\
\hline Facultad de Derecho & 46 & 13,5 & 13,5 & 51,5 \\
\hline Facultad de Economía & 55 & 16,1 & 16,1 & 67,5 \\
\hline Facultad de Medicina & 42 & 12,3 & 12,3 & 79,8 \\
\hline $\begin{array}{c}\text { Facultad de Medicina } \\
\text { Veterinaria }\end{array}$ & 32 & 9,4 & 9,4 & 89,2 \\
\hline Instituto Politécnico Superior & 37 & 10,8 & 10,8 & 100,0 \\
\hline Total & 342 & 100,0 & 100,0 & \\
\hline
\end{tabular}

\section{Confiabilidad de la escala}

Teniendo en cuenta la escala del cuestionario y según el coeficiente Alpha de Cronbach, utilizado como criterio de confiabilidad, Almeida, Santos y Costa (2010) apuntan que al existir un valor mayor o igual a 0.7 , como presentado en la tabla 4, la escala es confiable. Este resultado se obtuvo a través de los cálculos realizados en el SPSS Estatistic.

Tabla 5

Estadísticas de fiabilidad de escala de medición

\begin{tabular}{ll}
\hline Alpha de Cronbach & N. $^{\circ}$ de ítems \\
\hline 0,832 & 19 \\
\hline
\end{tabular}

\section{Caracterización del perfil de los estudiantes entrevistados}

Se encuestó a 342 estudiantes, 184 de ellos hombres, que representan el 53.8\%, y 158 mujeres, que representan el $46.2 \%$.

\section{Resultados}

Teniendo en cuenta el cuestionario aplicado y la propuesta presentada en el modelo de evaluación del desempeño del docente, elaborado y presentado junto al cuestionario, tratamos de identificar la opinión de los estudiantes sobre su validez y sobre su participación en el proceso de evaluación del desempeño. Esto permitió la elaboración de la propuesta de procedimiento para la evaluación del desempeño docente en la UJES. 


\section{Procedimiento propuesto para la evaluación del desempeño docente de la Universidade José Eduardo dos Santos}

El sistema de evaluación del desempeño diseñado y propuesto a la Universidade José Eduardo dos Santos involucra la participación de los estudiantes y combina el método de evaluación del desempeño de la competencia y el método de evaluación del desempeño de 360 grados. La combinación de estos dos métodos lo convierte en un método mixto, que permite al docente ser evaluado desde perspectivas de cuatro dimensiones, es decir, las habilidades que utilizan en su acción diaria durante el proceso de enseñanza-aprendizaje: las habilidades pedagógicas-didácticas, las habilidades de investigación, las habilidades de gestión académica y las habilidades de extensión universitaria. Con respecto al método de 360 grados, este permite que el docente sea evaluado desde todos los ángulos con los que interactúa, es decir, sus compañeros (colegas), el jefe inmediato, el docente mismo, teniendo especial atención a los estudiantes. Todos estos son elementos que juegan trascendental papel en términos de peso específico en el modelo propuesto.

El sistema es compuesto por tres fases y ocho etapas. En la primera fase integran las tres etapas: 1 - definición de estrategias y políticas de Recursos Humanos; 2 - objetivos del sistema de evaluación del desempeño; 3 - requisitos del sistema de evaluación del desempeño.

Este primer paso tiene como objetivo revelar los principales problemas existentes e identificar posibles alternativas para su solución, implicando:

a) Organización del equipo de trabajo - tiene la responsabilidad de aplicar el instrumento para evaluación del desempeño. Debe ser hecho por personas con sólidos conocimientos acerca de los recursos humanos (políticas y estrategias). Aquí los estudiantes de último año deben integrarse, siendo estos los mejores por tener conocimiento actualizado y comportamiento para el proceso;

b) Estado actual del proceso de Evaluación de desempeño en cada institución - aquí se denomina a los métodos y técnicas para recopilación de información de la institución, incluyendo análisis documental, observación directa, las encuestas y los cuestionarios. Además se determina la muestra a ser cuestionada (estudiantes).

c) Análisis de los resultados del diagnóstico del estado actual de proceso de evaluación del desempeño y propuestas de mejora.

d) Continuación - en función de los aspectos detectados se procura crear soluciones alternativas.

La segunda etapa (definición de los objetivos del sistema de evaluación de desempeño) es compuesta por:

\section{Objetivo general}

1. Su objetivo es mejorar y potenciar el papel del desempeño pedagógico, académico, de investigación y de extensión universitaria en la Universidade José Eduardo dos Santos a través de la participación de los estudiantes en el mismo proceso para identificar las debilidades en el desempeño del personal docente y su potencial de mejora;

2. Retroalimentar los otros procesos de Recursos Humanos vinculados a la evaluación de desempeño (selección, promoción, formación, recompensa y otros) y contribuir de manera sostenible a mejorar la calidad del proceso de enseñanza-aprendizaje.

\section{Objetivos específicos}

- Conocer las fortalezas y debilidades de los docentes para mejora de su desempeño; 
- $\quad$ Contribuir en la permanente actualización del cuerpo docente;

- Proporcionar a los docentes las opiniones de los distintos actores, con especial énfasis en los alumnos.

- Determinar los maestros que merecen premios y estímulos;

- Detectar la necesidad de formación y mediante contribuir a los planes de formación docente;

- Proporcionar cultura de evaluación de desempeño en un entorno de integración entre actores que ejercen la enseñanza con responsabilidad.

La tercera etapa se refiere a los requisitos del sistema. Lo mismo está diseñado para cumplir con 4 elementos desde el punto de vista de sus requisitos;

1. Objetividad - el proceso de evaluación del desempeño debe asociarse a la mejora de la calidad de la enseñanza y el desarrollo profesional de los docentes.

2. Imparcialidad - el proceso de evaluación del desempeño debe tener en cuenta el criterio de imparcialidad para que sea confiable, respetable y aceptable para los evaluadores.

3. Comunicación - el proceso de evaluación del desempeño debe ser fluido, tener características comunicativas, siendo que la comunicación debe existir para que el proceso fluya dialécticamente.

4. Evaluadores - el proceso de evaluación del desempeño debe ser desarrollado por personas creíbles respetadas por los evaluadores. Es necesario que los evaluadores puedan desempeñar sus funciones en un entorno de clara aceptación y reconocimiento de sus competencias y que los evaluados tengan plena confianza en los evaluadores.

\section{Fase II - Diseño del sistema de evaluación del desempeño}

Objetivo: explorar de manera detallada y secuencial cómo se estructura el diseño del sistema de evaluación del desempeño, que incluye la definición de métodos, indicadores de desempeño y la entrevista de evaluación del desempeño.

Cuarto paso: determinación de los métodos de evaluación del desempeño. El método propuesto mencionado anteriormente es un método mixto, resultado de la combinación del método de evaluación del desempeño de la competencia y el método de evaluación de 360 grados. La evaluación del desempeño de las competencias permite que tanto el evaluador como los superiores identifiquen qué competencias tiene el docente y cuáles deben mejorarse, expandirse o desarrollarse de una manera que coincida con las competencias esperadas por la institución educativa. A su vez, la evaluación de desempeño de 360 grados permite conocer la evaluación de los diferentes actores que se relacionan con los docentes, con especial énfasis en los estudiantes.

Quinta etapa - modelo de evaluación docente. Como se señaló anteriormente, la combinación del método de evaluación del desempeño por la competencia y el método de evaluación del desempeño de 360 grados para UJES tiene como objetivo lograr cuatro dimensiones de competencia: pedagógico-didáctico, de investigación, gestión académica y extensión universitaria. Teniendo en cuenta las percepciones de los estudiantes sobre las habilidades de evaluación, además de la validación del modelo en el anexo 1 para la evaluación del desempeño del estudiante, el instrumento se compone de 26 preguntas como se ilustra en el anexo 1. 
Sexta etapa - desarrollo de la entrevista de evaluación del desempeño. Una vez que se haya llevado a cabo la evaluación de desempeño, el docente será informado de su evaluación de acuerdo con los estudiantes, para mejorar su actualización futura. Este paso requiere que se sigan los siguientes pasos:

a) Preparación de la encuesta de evaluación del desempeño;

b) Contacto inicial con los docentes y creación de un entorno formal para la evaluación del desempeño;

c) Realización de la entrevista de evaluación del desempeño;

d) Informe acerca de la evaluación del desempeño;

e) Cierre de la entrevista de evaluación del desempeño.

\section{Fase III - Control del sistema de evaluación del desempeño}

Esta fase integra dos etapas que constituyen las etapas 7 y 8 . Esta fase está destinada a monitorear el sistema de evaluación del desempeño, asegurando que se respeten los objetivos, requisitos, métodos, indicadores establecidos y acciones de mejora del proyecto relacionadas con los docentes.

Séptima etapa - controles periódicos. Esta etapa se destina a la indicación de los fallos y problemas, así como sugestiones y soluciones existentes en el proceso de evaluación de desempeño por los estudiantes.

Octava etapa - monitoreo. Se destina a monitorear los resultados de control del sistema de evaluación de desempeño. Consiste en establecer revisiones periódicas para detectar posibles cambios estratégicos de la UJES referentes a evaluación de desempeño.

\section{Discusión y conclusiones}

El objetivo principal de esta investigación fue estudiar la gestión universitaria desde el punto de vista de la evaluación del desempeño docente, centrándose en la participación de los estudiantes en el proceso de evaluación, como una forma de contribuir a la mejora del desempeño docente y la calidad de la enseñanza en la Universidade José Eduardo dos Santos, además de proponer un procedimiento de evaluación del desempeño docente que integra su participación en el mismo proceso.

Como los estudiantes son la fuente principal para la evaluación del desempeño de los docentes, considerando que viven con ellos, aunque varios autores están de acuerdo y advierten el encuadre de los estudiantes en el proceso de evaluación del desempeño, se considera que tienen un mayor privilegio para emitir sus opiniones sobre el desempeño docente. Por lo tanto, en este trabajo, la evaluación del desempeño docente fue tratada como una de las estrategias de recursos humanos para la mejora de las políticas de desempeño y ejecución, entendiendo la necesidad que el mismo proceso trae a la mejora de la calidad de la enseñanza, la proyección y el desarrollo profesional del profesor.

Cuanto a las investigaciones realizadas, y con base en las encuestas respondidas por los estudiantes de las diferentes unidades orgánicas de la UJES, se considera que es necesario involucrar a los estudiantes en el proceso de evaluación de desempeño, teniendo en cuenta los objetivos de la Universidad, y basado en su acción, el modelo propuesto aquí toma en cuenta las cuatro dimensiones que se encajarían en la vida cotidiana de cada unidad orgánica. Aunque algunas U.O's hacen una evaluación de desempeño docente con base en el Decreto n. ${ }^{\circ} 25 / 94$, se pueden detectar algunas debilidades con respecto al proceso: 
- $\quad$ El proceso no es utilizado por todas las Unidades Orgánicas que componen la UJES;

- El proceso no tiene en cuenta la participación de los estudiantes como elemento principal de la enseñanza en el aula;

- $\quad$ El proceso no tiene en cuenta la evaluación de las competencias específicas del docente cuanto formador de ideas y considerando el trípode (enseñanza, investigación y extensión) de la acción de la Universidad;

- $\quad$ El proceso de evaluación no permite evaluar las cualidades docentes, teniendo en cuenta la evaluación formativa que garantiza el desarrollo profesional del profesor. De hecho, está más centrado en la evaluación sumativa que se utiliza por sí sola al tomar la decisión de promoción.

Así, esta investigación buscó, con la propuesta del procedimiento y el modelo de evaluación del desempeño docente, enmarcar a los alumnos, ya que su participación en el proceso puede contribuir al cambio de desempeño de los docentes, generando así una mejora en la calidad de la enseñanza y en la capacidad de gestión de la universidad, considerando que el modelo busca evaluar las competencias pedagógico-didácticas, teniendo en cuenta el desempeño y el proceso de enseñanza-aprendizaje, las competencias investigativas, considerando el grado de importancia que tiene la investigación en profesional docente, las habilidades de gestión académica teniendo en cuenta la participación del profesor en la gestión de la institución y, finalmente, las habilidades de extensión universitaria, como resultado de la práctica social del profesor. Todas estas habilidades que se buscan evaluar en los docentes están interconectadas, y por la investigación actual, los estudiantes tienen una alta percepción y consideran que estas habilidades deben ser evaluadas.

Siendo reciente en el contexto angoleño el tema de evaluación del desempeño de los docentes de educación superior, se recomienda a otros autores, junto con esta investigación, continuar con la misma, ya que eso trasciende su importancia capital, porque, si se aplica, puede catapultar las instituciones de educación superior con una alta calidad de enseñanza y aprendizaje a establecerse en el contexto regional africano y, por lo tanto, permitir que ocupen un lugar destacado en los rankings internacionales.

\section{Referencias}

Almeida, D., Santos, M. A. R., \&Costa, A. F. B. (2010). Aplicação do coeficiente alfa de Cronbach nos resultados de um questionário para avaliação de desempenho da saúde pública. Retrieved from http://www.abepro.org.br/biblioteca/enegep2010_TN_STO_131_840_16412.pdf.

Bohlander, G. \& Snell, S. (2009). Administração de Recursos Humanos.(14a Ed.). São Paulo:, Cengage Learning.

Camara, P. B. da, Guerra, P. B., \& Rodrigues, J. V. (2010). Novo Humanator, Recursos Humanos e Sucesso Empresarial. (3 ${ }^{\mathrm{a}}$ Ed.).Portugal: D.Quixote.

Carvalho, A.V. DE, Nascimento, L.P. do \& Serafim, O.C.G., (2012). Administração de Recursos Humanos. ( $2^{\mathrm{a}}$ Ed.).São Paulo: Cengage Learning.

Cassettari, N. (2014). Avaliação de docentes: uma questão de escolhas. Retrieved from http://publicacoes.fcc.org.br/ojs/index.php/eae/article/viewFile/2829/2702 
Chiavenato, I. (2009). Recursos Humanos. O capital humano das organizações. (9a Ed.).São Paulo: Elsevier Editora Ltda.

Daft, R.L. (2010). Administração.(2 ${ }^{\mathrm{a}}$ Ed.). São Paulo: Cengage Learning.

Decreto N. $25 / 94$, de 01 de Julho (1994). Estabelece as regras e procedimentos a observar na classificação dos funcionários públicos. Diário da República I Série, n. ${ }^{\circ} 26$.

Decreto N. ${ }^{0} 5 / 09$, de 7 de Abril (2009). Cria as regiões académicas que delimitam o âmbito territorial de actuação e expansão das instituições de ensino superior. Diário da República I Série, n. ${ }^{\circ} 64$.

Decreto N. ${ }^{9} 90 / 09$, de 15 de Dezembro (2009). Estabelece as normas gerais reguladoras do subsistema do ensino superior. Diário da República I Série, n. 237.

Decreto Presidencial N. ${ }^{2} 246 / 11$, de 14 de Setembro (2011). Aprova o Estatuto Orgânico da Universidade José Eduardo dos Santos. Diário da República I Série, n. ${ }^{\circ} 177$.

Drucker, P.F. (2010). Introdução à Administração, São Paulo.

Fernandes, D. (2008). Avaliação do desempenho docente: desafios, problemas e oportunidades. Retirado de: http://repositorio.ul.pt/bitstream/10451/5508/1/Brochura_avaliacao_docente.pdf.

Fernandes, D. J. (2014). El rol del estudiante en el proceso de evaluación del desempeño del docente universitario. El caso de la Universidad José Eduardo dos Santos. República de Angola. 8va. Conferencia Internacional de Ciências Empresariales.

Fernandes, D. J., Martinez, C. C. M. \& Sanchez, M. S (2015). Desempenho docente. Contribuição para o processo de integração dos estudantes que ingressam no ensino. Caso do Instituto Superior de Ciências da Educação do Huambo, República de Angola. Retrieved from http://revista.iscedhbo.ed.ao/index.php/rop/article/view/117/101.

Gomes, O. F., Gomide, T. R., Gomes, M. A. do N., De Araújo, D. C., Martins, S. \& FARONI, W. (2012). Sentidos e implicações da gestão universitária para os gestores universitários. In XIII Coloquio de Gestión Universitaria en Américas, Brasil. Retrieved from https://repositorio.ufsc.br/bitstream/handle/123456789/114881/2013121\%20$\% 20$ Sentidos $\% 20 \mathrm{e} \% 20 \mathrm{implica} \% \mathrm{C} 3 \% \mathrm{~A} 7 \% \mathrm{C} 3 \% \mathrm{~B} 5 \mathrm{es} \% 20 \mathrm{da} \% 20 \mathrm{gest} \% \mathrm{C} 3 \% \mathrm{~A} 3 \mathrm{o} \% 20 \mathrm{u}$ niversit $\% \mathrm{C} 3 \% \mathrm{~A} 1$ ria.pdf? sequence $=1$.

Pinto, J. P. \& Oliveira, E. R. (2005). Métodos de avaliação de desempenho dos docentes universitários. Retrieved from http//www.snesup.pt/cgi-bin

Rocha, E. P. (2004). Uma avaliação da eficiência do capital humano: feedback $360^{\circ}$. Retrieved from http://www.aedb.br/seget/arquivos/artigos $05 / 25$ feedback360graussimgen.pdf.

Sá, S. O., Alves, M. P., \& Costa, A. P. (2014). A avaliação formativa no ensino superior: o contributo do feedback interativo e construtivo na aprendizagem ativa dos estudantes. Retrieved from http://www.revistas.ufg.br/index.php/ci/article/view/31821/17611

Silverstein, B. (2011). Avaliação de desempenho. Aprenda a avaliar, promover e demitir. Rio de Janeiro: Senac Rio.

Tamo, K. (212) Metodologia de Investigação em Ciências Sociais, como elaborar um trabalho de fim de curso em gestão. (2 ${ }^{\mathrm{a}}$ Ed.). Luanda:Capatê-publicações, Lda. 
Tamo, K. (2006) Introdução à Gestão das Organizações, conceitos e estudos de casos. Luanda: Capatê-publicações, Lda.

Teixeira, S. (2013).Gestão das Organizações. (3 ${ }^{\text {a }}$ Ed.). Escolar Editora.

Tosta, H. T., Dalmau, M.B.L., Tosta, K.C.B.T., \& Tecchio, E.L. (2012). Gestores universitários: papel e Competências necessárias para o Desempenho de suas atividades nas Universidades federais, Brasil. Retrieved from https://periodicos.ufsc.br/index.php/gual/article/view/1983535.2012v5n2p1/22565

Universidade José Eduardo dos Santos (2015). Relatório anual 2014. Luanda: Mayamba Editora.

Fecha de recepción: 06/02/2019

Fecha de revisión: 18/05/2019

Fecha de aceptación: 27/08/2019 


\section{Anexo 1}

\section{Anexos}

Modelo propuesto para Evaluación de desempeño docente por el estudiante

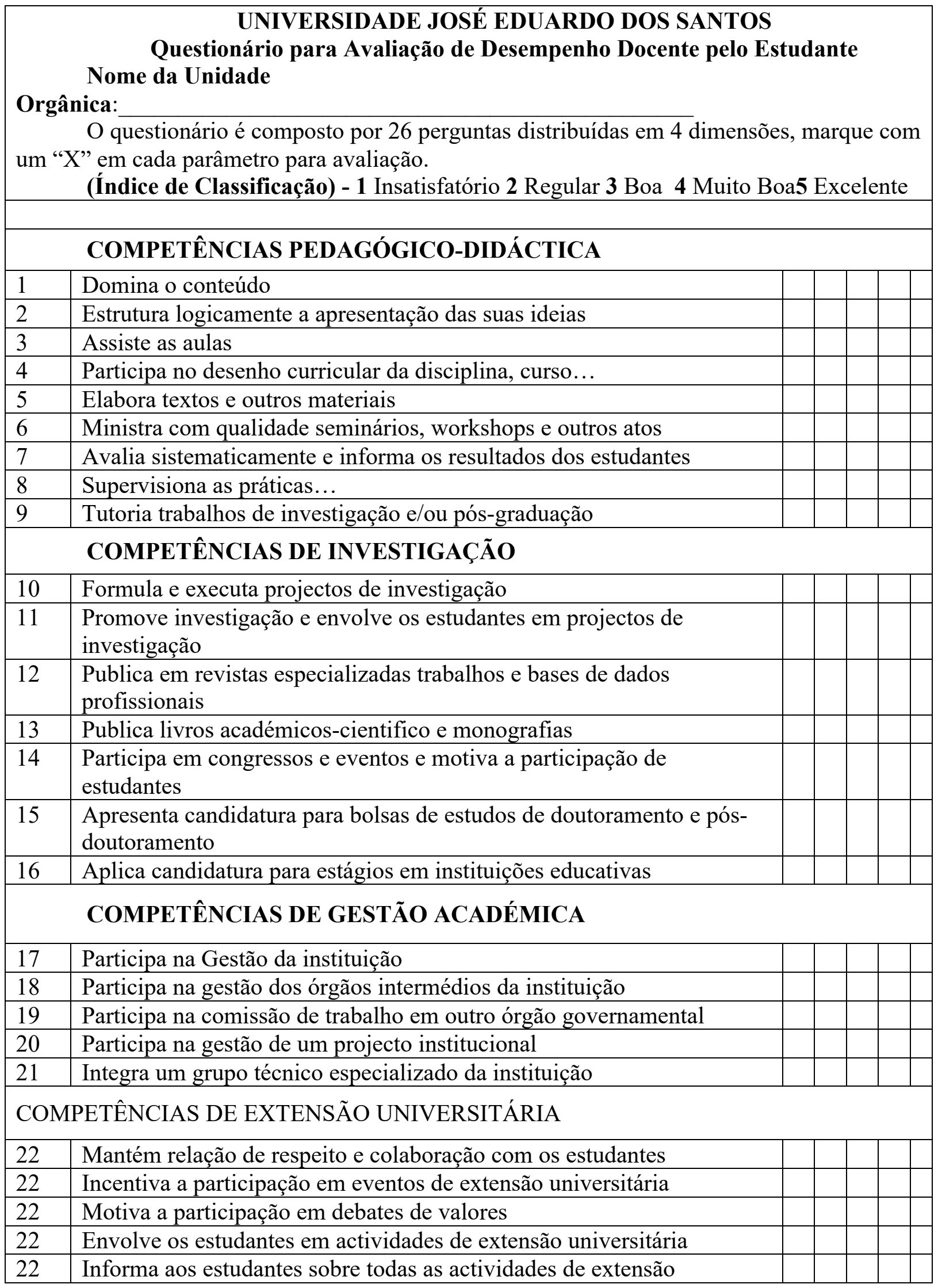




\begin{tabular}{|l|l|l|l|l|}
\hline universitária e projeção social & & & & \\
\hline \multicolumn{3}{|c|}{ OBSERVAÇ̃̃O } \\
\end{tabular}

Nota: O Autor, adptado de Fernandes (2014)

\section{Anexo 2}

\section{Cuestionario}

Estimado estudante, depois de analisar o questionário e o modelo de avaliação de desempenho do docente universitário proposto, queira por favor responder o instrumento abaixo que poderá contribuir para a validação do mesmo.

\section{I, Marque com um X os dados referentes ao seu perfil.}

\section{Instituição:}

Nome da Turma: $\quad N^{\circ}$ de estudantes da

turma

Sexo: $\quad$ Masculino; $\square$ Feminino.

Idade: $\square$ de 17 a 19 anos; $\square$ de 20 a 25 anos; $\square$ de 26 a 35 anos: $\square$ de 36 a 45 anos; $\square$ Mais de 45 anos.

Ano de escolaridade: $\square$ Primeiro; $\square$ Segundo; $\square$ rceiro; $\square$ uarto; $\square$ into; $\square$ to.

II Participastes da avaliação de desempenho docente no ano passado? $\quad \square ; \quad \square$,

Instrumento para validação dos Questionários de Avaliação de Desempenho Docente.

III Instruções.

Analise cada uma das afirmações presentes na tabela que se segue, coloque um círculo ao número que corresponde ao seu grau de concordância ou de discordância.

\begin{tabular}{|c|c|c|c|c|c|c|c|}
\hline & OPÇÕES DE RESPOSTAS & 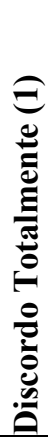 & 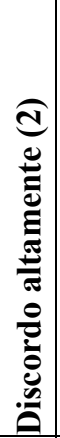 & 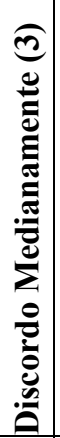 & 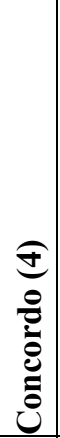 & 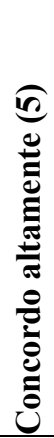 & 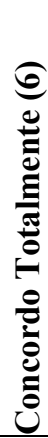 \\
\hline 1 & Os questionários dão resposta aos objectivos & 1 & 2 & 3 & 4 & 5 & 6 \\
\hline 2 & As questões apresentadas são suficientes para o propósito da investigação & 1 & 2 & 3 & 4 & 5 & 6 \\
\hline 3 & $\begin{array}{l}\text { Os questionários podem melhorar de forma integral a política de recursos } \\
\text { humanos da Universidade }\end{array}$ & 1 & 2 & 3 & 4 & 5 & 6 \\
\hline 4 & Os questionários são factíveis de aplicação em objectos de estudos práticos & 1 & 2 & 3 & 4 & 5 & 6 \\
\hline 5 & As perguntas constantes nos questionários são fluidas & 1 & 2 & 3 & 4 & 5 & 6 \\
\hline 6 & Os questionários são de extensão razoável & 1 & 2 & 3 & 4 & 5 & 6 \\
\hline 7 & Os questionários apresentam consistência lógica a partir da estrutura, & 1 & 2 & 3 & 4 & 5 & 6 \\
\hline
\end{tabular}




\begin{tabular}{|c|c|c|c|c|c|c|c|}
\hline & sequencia, inter-relação de aspectos e coerência metodológica interna & & & & & & \\
\hline 8 & Existem perguntas no questionário que devem ser eliminadas & 1 & 2 & 3 & 4 & 5 & 6 \\
\hline 9 & Existem perguntas em falta nos questionários que devem ser acrescidas & 1 & 2 & 3 & 4 & 5 & 6 \\
\hline 10 & A ordem das perguntas deve ser alterada para agilizar o fluxo das respostas & 1 & 2 & 3 & 4 & 5 & 6 \\
\hline 11 & A redação das questões deve ser melhorada & 1 & 2 & 3 & 4 & 5 & 6 \\
\hline 12 & $\begin{array}{l}\text { Os questionários e o modelo são extensíveis para outras universidades pela sua } \\
\text { capacidade de adaptabilidade, transparência, consistência e possibilidade de } \\
\text { generalização }\end{array}$ & 1 & 2 & 3 & 4 & 5 & 6 \\
\hline 13 & $\begin{array}{l}\text { Os questionários permitem diagnosticar e avaliar periódica e racionalmente o } \\
\text { desempenho docente }\end{array}$ & 1 & 2 & 3 & 4 & 5 & 6 \\
\hline 14 & Os questionários permitem detetar as principais debilidades dos docentes & 1 & 2 & 3 & 4 & 5 & 6 \\
\hline 15 & $\begin{array}{l}\text { Os questionários permitem que os diferentes atores que convivem com os } \\
\text { docentes possam oferecer as suas opiniões sobre o desempenho docente }\end{array}$ & 1 & 2 & 3 & 4 & 5 & 6 \\
\hline 16 & $\begin{array}{l}\text { Os questionários permitem melhorar as políticas e práticas de gestão de } \\
\text { recursos humanos e o melhoramento do desempenho laboral dos docentes }\end{array}$ & 1 & 2 & 3 & 4 & 5 & 6 \\
\hline 17 & $\begin{array}{l}\text { Os questionários possibilitam a percepção da situação estratégica dos recursos } \\
\text { humanos docentes na universidade }\end{array}$ & 1 & 2 & 3 & 4 & 5 & 6 \\
\hline 18 & $\begin{array}{l}\text { Com sua adoção e aplicação, consegue-se contribuir para o melhoramento } \\
\text { integral dos recursos humanos docentes }\end{array}$ & 1 & 2 & 3 & 4 & 5 & 6 \\
\hline 19 & $\begin{array}{l}\text { As competências laborais e os indicadores previstos nos questionários são } \\
\text { satisfatórios. }\end{array}$ & 1 & 2 & 3 & 4 & 5 & 6 \\
\hline
\end{tabular}

Marque em baixo o número correspondente aos questionários que sugere que seja retirada

\begin{tabular}{|l|l|l|l|l|l|l|l|l|l|l|l|l|l|l|l|l|l|l|}
\hline 1 & 2 & 3 & 4 & 5 & 6 & 7 & 8 & 9 & 10 & 11 & 12 & 13 & 14 & 15 & 16 & 17 & 18 & 19 \\
\hline & & & & & & & & & & & & & & & & & & \\
\hline
\end{tabular}

\section{Caso queira que alguma pergunta seja acrescentada aos questionários, escrevê-la no quadro que se segue}

Com base nas respostas referentes as 4 categorias de competências mencionadas na escala (competências pedagógica-didática, competência investigativa, competência de gestão académica e competência de extensão), mencione como percebe o impacto de cada uma delas no desempenho docente: coloque um círculo sobre os números 1,2,3, 4 ou 5, que melhor represente a sua percepção.

\begin{tabular}{|l|l|l|l|l|l|}
\hline $\begin{array}{l}\text { I Competências } \\
\text { Pedagógica- } \\
\text { didática }\end{array}$ & Muito Baixo & Baixo & Médio & Alto & Muito Alto \\
\cline { 2 - 7 } & 1 & 2 & 4 & 4 & 5 \\
\hline $\begin{array}{l}\text { II Competências } \\
\text { Investigativa }\end{array}$ & Muito Baixo & Baixo & Médio & Alto & Muito Alto \\
\cline { 2 - 6 } & 1 & 2 & 3 & 4 & 5 \\
\hline $\begin{array}{l}\text { III Competências } \\
\text { de Gestão } \\
\text { Académica }\end{array}$ & Muito Baixo & Baixo & Médio & Alto & Muito Alto \\
\cline { 2 - 6 } & 1 & 2 & 3 & 4 & 5 \\
\hline $\begin{array}{l}\text { IV Competências } \\
\text { de Extensão } \\
\text { Universitária }\end{array}$ & Muito Baixo & Baixo & Médio & Alto & Muito Alto \\
\cline { 2 - 6 } & 1 & 2 & 3 & 4 & 5 \\
\hline
\end{tabular}


Gestión universitaria. Contribución del estudiante para el proceso de evaluación de desempeño docente, aplicación en la Universidad José Eduardo dos Santos, Huambo-Angola

Anexo 3

Modelo de Evaluación de la Función Pública Angoleña

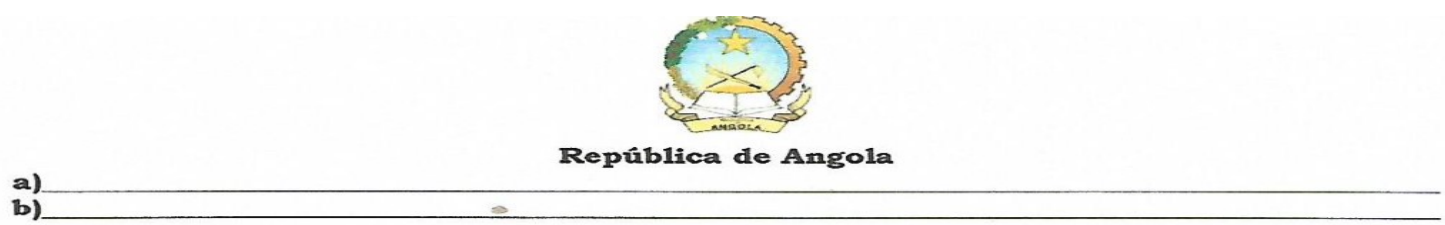

NOTAC̣ÃO ANUAL DO PESSOAL TÉCNICO SUPERIOR, DO PESSOAI TÉCNICO E TÉCNICO MIÉDIO

NOME:

CATEGORIA:

DATA DA NOTAÇÃO

CLASSIFICAÇÃO DE SERVIÇO

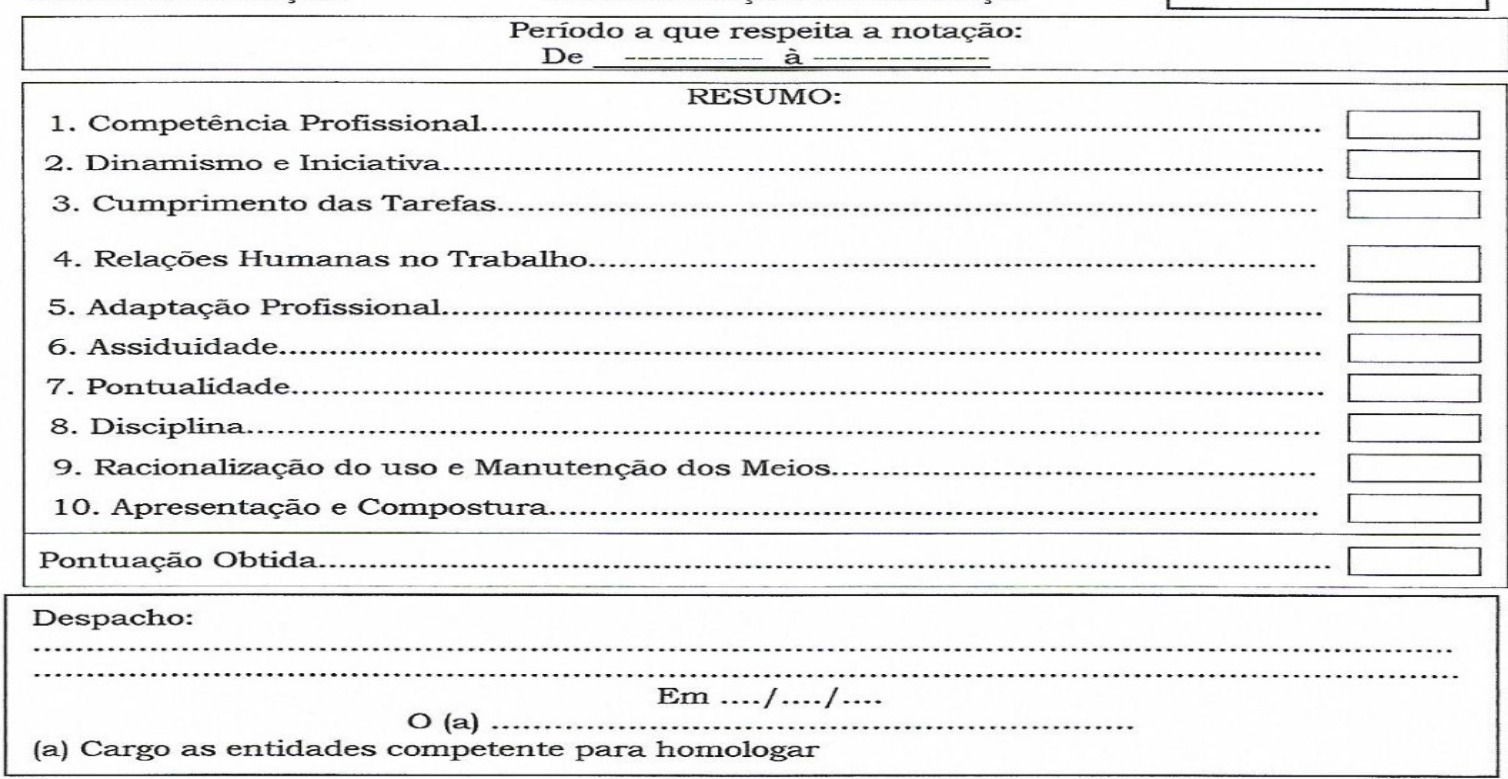

Tomei conhecimento após homologação

O NOTADO

$\operatorname{Em} \ldots . . . . . / \ldots$.

$$
\begin{aligned}
& \text { PRÉ EQUAÇÃO } \\
& \mathrm{C}=\mathrm{g}+\frac{\mathrm{Sn}-\mathrm{In}}{\mathrm{N} \times \mathrm{n}}
\end{aligned}
$$

Pontuação Final obtida

C - Pontuaçāo Final após pré equaçāo g - Pontuação obtida pelo notado

S - Somatōrio das pontuações obtidas por todos os funcionários e agentes dos serviços ou organismos que têm a mesma categoria do notado.

I - Somatório das pontuações obtidas pelos funcionários e agentes de idêntica categoria, atribuidas pelos notadores que avaliaram o notado.

N - Número total de funcionários e agentes pertencentes à categoria do notado.

n - Nümero de funcionários e agentes da mesma categoria apurados pelos notadores que avaliaram o notado. 


\section{O NOTADO}

Tomei conhecimento:

$\operatorname{Em} \ldots \ldots / \ldots \ldots / \ldots \ldots$

\section{OS NOTADORES}

$\operatorname{Em} \ldots \ldots / \ldots \ldots / \ldots \ldots$

$\operatorname{Em} \ldots \ldots / \ldots \ldots / \ldots \ldots$

(a) Ministério ou Secretaria de Estado

(b) Órgão a que pertence o notado.

FUNÇÕES EXERCIDAS DURANTE O PERÍODO EM APRECIAÇÃO

\begin{tabular}{|c|c|c|}
\hline ENTIDADE & FUNÇÕES EXERCIDAS & PERIOODO \\
\hline & & \\
\hline & & \\
\hline
\end{tabular}

\section{ANTIGUIDADE}

De Serviço

Na Carreira

$\mathrm{Na}$ Categoria

\section{ACTIVIDADES RELEVANTES DURANTE O PERÍODO EM APRECIAÇÃO}

\begin{tabular}{|c|c|c|}
\hline FORMAÇĀO & TRABALHOS & MISSŌES ESPECÍFICAS \\
\hline
\end{tabular}

\section{APRECIAÇĀO GERAL}

(A PREENCHER PELOS NOTADORES)

1. Apreciação geral salientando se há ou não adaptação à função, quais os pontos fortes e fracos e quais os meios de aperfeiçoamento adequados:

2. Opinião sobre a aptidāo do notado para o eventual exercício de funçōes de categoria superior e de função de chefia: 